\title{
Pulmonary HIV/AIDS-associated sarcoidosis
}

\section{Editorial}

The majority of cases with human immunodeficiency virus (HIV)/ acquired immunodeficiency syndrome (AIDS) associated pulmonary sarcoidosis have been related to immune reconstitution secondary to highly active antiretroviral therapy (HAART). Some previous studies demonstrated association between worsening of sarcoidosis course and CD4+ T lymphocyte count higher than 200cells $/ \mathrm{mm}^{3}$, as well as association between the improvement of clinical symptoms and CD4+ T-cell count less than 200cells $/ \mathrm{mm}^{3}$. Nevertheless, the onset of pulmonary sarcoidosis in HIV-infected/AIDS patients was demonstrated before the HAART era. The majority of the patients reveal restrictive lung pattern, but obstructive lung pattern can be identified. Bronchoalveolar lavage (BAL) demonstrates significantly high eosinophil, neutrophil, and lymphocyte counts, including significantly high CD4+: CD8+ T-cell ratio. BAL CD4+: CD8+ T-cell ratio is also significantly higher than the ratio in the blood at the time of diagnosis. Interleukin-2 or interferon- $\alpha 2$ a may be associated with pulmonary sarcoidosis development. The course of pulmonary sarcoidosis is similar in both HIV-infected/AIDS and HIVseronegative patients. There is no significantly different change in virologic and immunologic parameters between patients with HAART in whom pulmonary sarcoidosis is clinically and radiologically cured or improved and pulmonary sarcoidosis patients with HAART that deteriorated or remained stable.

In conclusions, HIV-infected/AIDS patients with long-term immunologic reconstitution during HAART can develop pulmonary sarcoidosis. BAL-fluid analysis findings, clinical and radiological findings, and outcome are similar in both HIV-infected/AIDS and HIV-seronegative patients.

\section{Acknowledgements}

None.

\section{Conflict of interest}

The author declares no conflict of interest.
Volume 4 Issue I - 2017

\author{
Attapon Cheepsattayakorn, ${ }^{1,2,3}$ Ruangrong \\ Cheepsattayakorn ${ }^{4}$ \\ 'Editor-in-Chief, Journal of Lung, Pulmonary and Respiratory \\ Research, USA \\ ${ }^{2}$ IOth Zonal Tuberculosis and Chest Disease Center, Chiang Mai, \\ Thailand \\ ${ }^{3}$ I Oth Office of Disease Prevention and Control, Chiang Mai, \\ Department of Disease Control, Ministry of Public Health, \\ Thailand \\ ${ }^{4}$ Department of Pathology, Faculty of Medicine, Chiang Mai \\ University, Chiang Mai, Thailand
}

Correspondence: Attapon Cheepsattayakorn, I0th Zonal Tuberculosis and Chest Disease Center, I 43 Sridornchai Road Changklan Muang Chiang Mai 50100 Thailand,Tel 6653 140767, 6653 276364, Fax 6653 140773, 6653273590 ,

Email Attapon 1958@gmail.com , attaponche@yahoo.com

Received: January 18, 2017 | Published: January 26, 2017 